\title{
Correction to: Analysis of glutathione S-transferase and cytochrome P450 gene polymorphism in recipients of dose-adjusted busulfan-cyclophosphamide conditioning
}

\author{
Seitaro Terakura ${ }^{1,2}$ - Makoto Onizuka ${ }^{1,3} \cdot$ Mariko Fukumoto $^{4}$ - Yachiyo Kuwatsuka ${ }^{1,5} \cdot$ Akio Kohno $^{6}$. \\ Yukiyasu Ozawa ${ }^{1} \cdot$ Koichi Miyamura $^{1} \cdot$ Yuichiro Inagaki $^{7} \cdot$ Masashi Sawa $^{7} \cdot$ Yoshiko Atsuta $^{1,8} \cdot$ Ritsuro Suzuki $^{8}$.

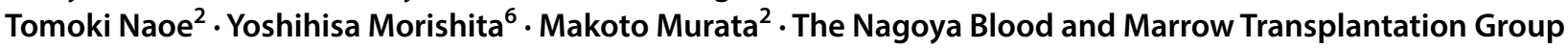

Published online: 26 November 2019

(c) Japanese Society of Hematology 2019

\section{Correction to: International Journal of Hematology https://doi.org/10.1007/s12185-019-02741-8}

In the original article of Terakura et al., the COI disclosure were missing. The corrected disclosure statement is as follows:

S. Terakura reports personal fees from Chugai Pharmaceutical Co., Ltd., Yakult Honsha Co., Ltd., Otsuka Pharmaceutical Co., Ltd., Sumitomo Dainippon Pharma, Astellas Pharma Inc., Novartis, and Amgen Astellas BioPharma K. K., outside the submitted work; M. Onizuka reports grants

The original article can be found online at https://doi.org/10.1007/ s12185-019-02741-8.

Seitaro Terakura

tseit@med.nagoya-u.ac.jp

1 Department of Hematology, Japanese Red Cross Nagoya First Hospital, Nagoya, Japan

2 Department of Hematology and Oncology, Nagoya University Graduate School of Medicine, 65 Tsurumai-cho, Showa-ku, Nagoya 466-8550, Aichi, Japan

3 Department of Hematology and Oncology, Tokai University School of Medicine, Isehara, Japan

4 Division of Clinical Toxicology, Research and Education Center for Clinical Pharmacy, School of Pharmaceutical Sciences, Kitasato University, Tokyo, Japan

5 Department of Advanced Medicine, Nagoya University Hospital, Nagoya, Japan

6 Department of Hematology and Oncology, JA Aichi Konan Kosei Hospital, Konan, Japan

7 Department of Hematology and Oncology, Anjo Kosei Hospital, Anjo, Japan

8 Department of HSCT Data Management/Biostatistics, Nagoya University Graduate School of Medicine, Nagoya, Japan from Chugai Pharmaceutical Co., Ltd., personal fees from Bristol-Myers Squibb, Pfizer Inc., Sumitomo Dainippon Pharma Co., Ltd., Astellas Pharma Inc., Novartis, Asahi Kasei Pharma, and Daiichi-Sankyo, Inc., outside the submitted work; A. Kohno reports personal fees from Celgene Corporation, Novartis Pharma K. K., Bristol-Myers Squibb, Chugai Pharmaceutical Co., Ltd., Otsuka Pharmaceutical Co., Ltd., Takeda Pharmaceutical Co., Ltd., Ono Pharmaceutical Co., Ltd., and Janssen Pharmaceutical K. K., outside the submitted work; Y. Ozawa reports personal fees from Pfizer Japan, Astellas Pharma, Novartis Pharma, and Kyowa Hakko Kirin, outside the submitted work; K. Miyamura reports personal fees from Bristol-Myers Squibb, Novartis, Pfizer Japan Inc., Otsuka Pharmaceutical, Takeda Pharmaceutical, Kyowa-Hakko Kirin Co., Ltd., Chugai Pharmaceutical Co., Ltd., Astellas Pharma Inc., and Celgene, outside the submitted work; Y. Inagaki reports personal fees from Celgene, Takeda Pharmaceutical Co., Ltd., Mundipharma K.K., Bristol-Myers Squibb, Eisai Co., Ltd., Novartis Pharma K.K., and Kyowa-Hakko Kirin Co., Ltd., outside the submitted work; M. Sawa reports personal fees from Chugai, Pfizer, Astellas, Nippon-Shinyaku, Ono, MSD, BristolMyers Squibb, Kyowa-Hakko Kirin, Asahi-Kasei, Novartis, Eisai, Otsuka, Sumitomo Dainippon, Sanofi, Takeda, Celgene, Mochida, Shire, and Mundipharma, outside the submitted work; Y. Atsuta reports personal fees from Mochida Pharmaceutical Co., Ltd., Chugai Pharmaceutical Co., Ltd., Kyowa-Hakko Kirin Co., Ltd, Bristol-Myers Squibb Co., Ltd, Yakult Honsha Co., and Ltd, Janssen Pharmaceutical K.K., outside the submitted work; R. Suzuki reports personal fees from Bristol-Meyer Squib, Novartis, KyowaHakko Kirin, Chugai Pharmaceuticals, Shionogi, Takeda, Meiji Seika Pharma, MSD, Otsuka, Sawai, Celgene, Sumitomo Dainippon, Eisai Pharmaceuticals, Alexion Pharma, Sanofi, Gilead Sciences, Abbvie Inc., Mundi Pharma, Jazz 
Pharma, Ono Pharma, and Janssen Pharmaceuticals, outside the submitted work; Y. Morishita reports personal fees from Celgene corporation, outside the submitted work; M. Murata reports personal fees from Chugai Pharmaceutical Co., Ltd., GSK Co., Ltd., Otsuka Pharmaceutical Co., Ltd., Sumitomo Dainippon Pharma, Astellas Pharma Inc., Novartis, MSD
Co., Ltd., Kyowa-Hakko Kirin Co., Ltd., Bristol-Myers Squibb, Ltd., JCR Pharmaceuticals Co., Ltd., and Celgene Co., Ltd., outside the submitted work; the other authors have nothing to disclose. 